\title{
Treatment of Sensitive Tuberculosis: Mechanisms of Action and Resistance
}

\author{
Aline dos Santos Peixoto, Romário Martins Araújo, Haiana Charifker Schindler and Lilian Maria Lapa \\ Montenegro Pimentel* \\ Department of Immunology, Aggeu Magalhães Institute, Oswaldo Cruz Foundation, Brazil \\ *Corresponding author: Lilian Maria Lapa Montenegro Pimentel, Department of Immunology, Aggeu Magalhães Institut€
} Oswaldo Cruz Foundation, Recife, PE, Brazil

\section{ARTICLE INFO \\ Received: May 16, 2019 \\ Published: 㓞 June 04, 2019 \\ Citation: Aline dos Santos P, Romário Martins A, Haiana Charifker S, Lilian Maria Lapa Montenegro P. Treatment of Sensitive Tuberculosis: Mechanisms of Action and Resistance. Biomed J Sci \& Tech Res 18(4)-2019. BJSTR.} MS.ID.003174.

Keywords: Tuberculosis; Mycobacterium Tuberculosis; Antituberculosis Drugs; Drug Resistance; Mechanisms; Multidrug-Resistant Tuberculosis; Extensively Drug-Resistant Tuberculosis; Isoniazid; Rifampin; Pyrazinamide; Etambutol
ABSTRACT

The tuberculosis (TB) is a disease caused by Mycobacterium tuberculosis (Mtb) and is considered a worldwide public health problem, classified as the leading cause of death by a single infectious agent. The conventional treatment is performed through the combination of drugs that exhibit precocious or sterilizing bactericidal activity. The commonly used drugs (knowing as first line drugs) are: izoniazide (INH), rifampicin (RIF), pyrazinamide (PZA) and ethambutol (ETH). However, the number of resistant TB cases (classified as RR-TB, MDR-TB and XDR-TB) to drugs has been growing in concern over the years. Of the total cases registered in 2016, about 600 thousand were resistant to one or more drugs used in the treatment. Resistance of Mtb strains to antituberculosis drugs is closely related to mutations in different bacillus genes. Multidrugresistant tuberculosis is a worldwide problem. A better understanding of the molecular mechanisms associated with the resistance to first-line drugs used in the TB treatment is of great importance for the development of new drugs, directly helping to reduce the number of resistant cases and bringing great benefits to public health. A review of the mechanisms of action of first-line drugs used in the treatment regimen to sensitive tuberculosis, as well as the main associated resistance mechanisms are.

Abbreviations: TB: Tuberculosis; MTB: Mycobacterium Tuberculosis; RIF: Resistant to Rifampicin; PZA: Pyrazinamide; ETH: Etambutol; WHO: World Health Organization; ANTI-TB: Anti-Tuberculosis

\section{Introduction}

Tuberculosis (TB) is an infectious disease of chronic evolution caused by Mycobacterium tuberculosis (Mtb) [1]. Considered a priority public health problem, TB remains one of the top 10 causes of illness and death in the world population. In 2017, an estimated 10 million new TB cases worldwide were estimated with approximately 1.3 million deaths [2]. However, according to WHO estimates, these numbers are far from realistic [3]. The use of inadequate treatment regimens is frequent, being directly associated with the increase in cases of drug resistant TB $[4,5]$. Of the total number of cases registered in 2016, approximately 600,000 were believed to be resistant to rifampicin (RIF), of which 490,000 were resistant to both isoniazid (INH) and RIF and were classified as multidrug resistant (MDR-TB) [6,7]. Extensively drug resistant tuberculosis (XDR-TB) is characterized as a MDR-TB that is resistant to fluoroquinolones and second-line injectable drugs (amikacin, capreomycin and kanamycin) responsible for $6.2 \%$ of the incidence of resistant TB cases [6,7]. The treatment of tuberculosis is carried out through the association of drugs that have bactericidal or pre-sterilizing activity. The commonly used drugs (first-line drugs) are INH, RIF, etambutol (ETH) and pyrazinamide (PZA) for the twomonth period, followed by another four months with INH and RIF [8]. However, for resistant TB cases, treatment may last from 18 to 24 months, requiring the use of injectable medication, which may lead to the development of several side effects in patients $[6,8]$. Further elucidation of the mechanisms involved in the drug resistance process commonly used in the treatment of TB would help in the development of new drugs, directly assisting in reducing the number of resistant cases, bringing great benefits to public health. 


\section{Mechanisms of Drug Resistance in Mycobacterium Tu- berculosis}

Proper drug association, correct dosage, and long-term use are the basic principles for treatment, avoiding bacterial persistence and developing drug resistance, and thus ensuring patient healing. Currently, the standard treatmentrecommended by the World Health Organization (WHO) is the concomitant use of four antimicrobials: isoniazid, ethambutol, rifampicin and pyrazinamide given once in a single dose [2]. Among the characteristics of mycobacteria, the cell wall contains virulence factors that alter the normal functioning of host cells. The lipid coating imparts the group's distinguishing characteristics as acid resistance, extreme hydrophobicity and resistance to poor disinfectants. It probably also contributes to the slow growth rate of some species by restricting nutrient uptake [9]. Such impermeability of the envelope justifies the presence of pore-forming proteins (porins) as well as making it difficult for an effective host response to combat this pathogen [10].

The cases of resistant tuberculosis are classified according to the sensitivity of $\mathrm{M}$. tuberculosis to the first- and second-line medications used in the drug treatment of disease. The presence of multidrug resistance (resistance to RIF / INH or another firstline drug) indicates the use of the treatment regimen for MDR-TB, which involves second-line treatment drugs. Extensively resistant tuberculosis treatment should be performed in a tertiary health care network, which specializes in the treatment of resistant tuberculosis. Individualized schedules and reserve drugs are used [11]. It is worth noting that the treatment of the resistant forms becomes longer, exceeding 6 months of the traditional scheme and can reach up to 24 months or more [12].

\section{Mechanism of Action and Resistance to First-Line Treatment Drugs}

Isoniazid: The hydrazide of isonicotinic acid or isoniazid (INH), due to its significant bactericidal activity, has become a fundamental component of first-line anti-tuberculosis (ANTI-TB) regimens [13]. It was inserted into the anti-TB regime in the 50's which remains to this day a four-drug regime implemented globally for susceptible TB drugs [14]. The prodrug INH enters the cytoplasm of $\mathrm{M}$. tuberculosis through simple passive diffusion; being able to act only on microorganisms in the active process of replication, not acting during the stationary phase of growth or growing under anaerobic conditions $[14,15]$. Because it is a prodrug, INH is activated by the enzyme catalase-peroxidase encoded by the KatG gene. Once activated it interferes in the synthesis of essential mycolic acids by inhibiting NADH-enoyl-ACP-reductase, which is encoded by the inhA gene $[13,16]$. Mutations in the katG gene play a very important role in mediating resistance to INH as it develops the inactivation of this non-essential enzyme $[15,17]$.

Etambutol: Etambutol (ETH) was inserted into the treatment of tuberculosis in the 1960s, acting against intra- and extracellular bacilli, with a bacteriostatic characteristic [11]. ETH and its mechanism of action involves several cellular activities; the most likely mechanism is that the drug acts as an inhibitor of arabinosyl transferase enzymes involved in cell wall biosynthesis $[18,19]$. It is also known that ethambutol has bacteriostatic activity against non-replicating bacteria, bactericidal against replicating bacilli, as well as intramacrophagic activity [20]. The resistance mechanism of ETH is mainly linked to missense mutations in the embrB gene. The mutation of codon $306 \mathrm{embB}$ is more frequent in clinical isolates resistant to EMB, representing up to $68 \%$ of resistant strains [16]. To confirm ETH resistance, the region that determined this resistance in strains spontaneously resistant to ethambutol was sequenced. The results suggested embrB 306 mutations are sufficient to confer resistance to ethambutol and the detection of such mutations should be considered in the development of rapid molecular tests [14]. Recently, mutations in the ubiA gene, which synthesize enzymes involved in cell wall synthesis, have also been characterized as causing ethambutol resistance in conjunction with mutations in the embrB gene [16].

Rifampicin: Rifampin (RIF), a semi-synthetic derivative of rifamycin, was first introduced as a first-line drug against tuberculosis in the 1970s and is currently still considered one of the most potent drugs in the treatment of M. tuberculosis [21,22]. RIF diffuses freely through the M. tuberculosis cell wall and once inside the cell, inhibits gene transcription, with the blocking of the RNA polymerase enzyme, preventing the transcription of messenger RNA [21]. Resistance to RIF in M. tuberculosis can be attributed mainly to $\beta$-subunit modifications of RNA due to mutations in rpoB gene coding. Mutations in the $81 \mathrm{bp}$ region of rpoB gene are found in about $96 \%$ of RIF-resistant M. tuberculosis isolates. Mutations at positions 531, 526 and 516 are among the most frequent mutations in RIF-resistant lines [21\&13]. We highlight that although RIF interferes in the synthesis of RNA by binding to the $ß$-subunit of RNA polymerase, it was evidenced that RIF binding to the rpoB target induced hydroxyl radical formation in susceptible but nonresistant bacilli [16].

Pyrazinamide: Pyrazinamide (PZA), a nicotinamide analogue, which is the precursor of niacin (vitamin B3 and nicotinic acid), is the only drug in the current TB treatment regimen with sterilizing activity [23]. Unlike all other drugs involved in the treatment of TB, the PZA has a better action against bacilli that are in slow multiplication activity [16]. PZA plays a key role in the treatment of first-line tuberculosis, as it contributes to the reduction of treatment time from 12 to 6 months [21]. It is known that PZA is a prodrug that crosses the membrane of the Mycobacterium by passive diffusion and that in the cytoplasm is transformed into pyrazinoic acid (POA) by activity of the enzyme pyrazinamidase/ nicotinamidase encoded by the pncA gene of M. tuberculosis [16]. The POA causes the intracellular medium to become acid causing alterations in the permeability and transport characteristics of 
the membrane, provoking cellular damage besides interacting with enzymes linked to the translation phase of protein synthesis, inhibiting its synthesis $[13,23]$. Its mechanism of action against $M$. tuberculosis is the least understood of all first-line tuberculosis drugs [21]. The conventional model proposed that a low $\mathrm{pH}$ was required for PZA activity, but this was questioned suggesting that PZA also fatty acid synthesis, which is not yet well characterized. Other hypotheses would be that the PZA could block some virulence factor of the bacterium or that the PZA may have the ability to bind to the ribosomal protein S1 (RpsA), which interrupts the translation of the protein [24]. Mutations in the pncA gene are the main mechanism of resistance of PZA in M. tuberculosis. PncA mutations are highly diverse and spread throughout the gene, which is unique to PZA resistance. Most PZA-resistant strains of M. tuberculosis (72-99\%, mean 85\%) have mutations in pncA [16].

\section{Conclusion}

The current treatment regimen is still long, totaling 6 months to complete, when success is achieved. This long period compromises the adherence of the patient, who often ends up abandoning treatment due to the time and related adverse effects, mainly the hepatotoxicity caused by all antimicrobials. Because of the low availability of medicines on the market and the failures to cure this disease due to abandonment or errors in administration, patients with multidrug-resistant bacilli appeared on first-line drugs and, in some cases, on second-line drugs. Obtaining new drugs with various pharmacological potentials becomes fundamental and increasingly necessary due, among other reasons, to the resistance of microorganisms to drugs.

\section{Acknowledgement}

We thank the staff of the Laboratory of Immunoepidemiology, Department of Immunology, Aggeu Magalhães Institute, Oswaldo Cruz Foundation.

\section{References}

1. Cole ST, Brosch R, Parkhill J, Garnier T, Churcher C, et al. (1998) Deciphering the Biology of Mycobacterium tuberculosis from the complete genome sequence. Nature 393(6685): 537-544.

2. (2017) Global tuberculosis report. Geneva: World Health Organization.

3. Jamal FJ, Moherdaui F (2007) Tuberculosis and HIV infection in Brazil: magnitude of the problem and strategies for control. Rev Saude Publica 41(1): 104-110.

4. Bailey SL, Roper MH, Huayta M, Trejos N, López Alarcón V, et al. (2011) Missed opportunities for tuberculosis diagnosis. The international journal of tuberculosis and lung disease 15(2): 205-210.

5. Walker IF, Shi O, Hicks J, Elsey H, Wei X, et al. (2019) Analysis of loss to follow up in 4,099 multidrug-resistant pulmonary tuberculosis patients. European Respiratory Journal: 53(5).

6. Ferreira KR, Orlandi GM, Silva TC, Bortolozzi MR, França FOS, et al. (2018) Representações sobre a adesão ao tratamento da Tuberculose Multidroga Resistente. Rev Esc Enferm USP 52: e03412.
7. Koch A, Cox H, Mizrahi V (2018) Drug-resistant tuberculosis: challenges and opportunities for diagnosis and treatment. Current opinion in pharmacology 42: 7-15.

8. (2011) BRASIL. MINISTÉRIO DA SAÚDE. SECRETARIA DE VIGILÂNCIA EM SAÚDE. DEPARTAMENTO DE VIGILÂNCIA EPIDEMIOLÓGICA. Manual de recomendações para o controle da tuberculose no Brasil.

9. Saravanan M, Niguse $S$, Abdulkader M, Tsegay E, Hailekiros $H$, et al. (2018) Review on emergence of drug-resistant tuberculosis (MDR \& XDR-TB) and its molecular diagnosis in Ethiopia. Microbial pathogenesis, 117: $237-242$

10. Daffé M. (2015) The cell envelope of tubercle bacilli. Tuberculosis 95: S155-S158.

11. Rabahi MF, Silva Junior LR, Ferreira ACG, Silva DGST, Conde MB, et al. (2017) Tratamento da tuberculose. Jornal Brasileiro de Pneumologia 43(5): 472-486.

12. Horsburgh CR Jr, Barry CE 3rd, Lange C (2015) Treatment of tuberculosis. New England Journal of Medicine, 373(22): 2149-2160.

13. Chakraborty Sumit, Rhee Kyu Y (2015) Tuberculosis drug development: history and evolution of the mechanism-based paradigm. Cold Spring Harbor perspectives in medicine 5 (8): a021147.

14. Bhat ZS, Rather MA, Maqbool M, Lah HU, Yousuf SK, et al. (2017) Cell wall: a versatile fountain of drug targets in Mycobacterium tuberculosis. Biomedicine \& Pharmacotherapy 95: 1520-1534.

15. Unissa AN, Subbian S, Hanna LE, Selvakumar N (2016) Overview on mechanisms of isoniazid action and resistance in Mycobacterium tuberculosis. Infection Genetics and Evolution 45: 474-492.

16. Zhang Y, YewWW (2015) Mechanisms of drug resistance in Mycobacterium tuberculosis: update 2015. The International Journal of Tuberculosis and Lung Disease 19 (11): 1276-1289.

17. Hoagland DT, Liu J, Lee RB, Lee RE (2016) New agents for the treatment of drug-resistant Mycobacterium tuberculosis. Advanced drug delivery reviews 102: 55-72.

18. Zimmerman M, Lestner J, Prideaux B, O'Brien P, Dias-Freedman I, et al. (2017). Ethambutol partitioning in tuberculous pulmonary lesions explains its clinical efficacy. Antimicrobial agents and chemotherapy 61(9): e00924-17.

19. Larsen EM, Stephens DC, Clarke NH, Johnson RJ (2017) Ester-prodrugs of ethambutol control its antibacterial activity and provide rapid screening for mycobacterial hydrolase activity. Bioorganic \& medicinal chemistry letters 27(19): 4544-4547.

20. Chamberlain PD, Sadaka A, Berry S, Lee AG (2017) Ethambutol optic neuropathy. Current opinion in ophthalmology 28 (6): 545-551.

21. Du Preez I, Loots DT (2018) Novel insights into the pharmacometabonomics of first-line tuberculosis drugs relating to metabolism, mechanism of action and drug-resistance. Drug metabolism reviews 50 (4): 466-481.

22. Tuyiringire N, Tusubira D, Munyampundu JP, Tolo CU, Muvunyi CM, et al. (2018) Application of metabolomics to drug discovery and understanding the mechanisms of action of medicinal plants with antituberculosis activity. Clinical and translational medicine 7(1): 29.

23. Lambert N, Abdalla AE, Duan X, Xie J (2017) Emerging drugs and drug targets against tuberculosis. Journal of drug targeting 25(4): 296-306.

24. Caño-Muñiz S, Anthony R, Niemann S, Alffenaar JC (2018) New approaches and therapeutic options for Mycobacterium tuberculosis in a dormant state. Clinical microbiology reviews 31(1): e00060-17. 
ISSN: 2574-1241

DOI: 10.26717/BJSTR.2019.18.003174

Lilian Maria Lapa Montenegro P. Biomed J Sci \& Tech Res

(C) (i) This work is licensed under Creative

Submission Link: https://biomedres.us/submit-manuscript.php

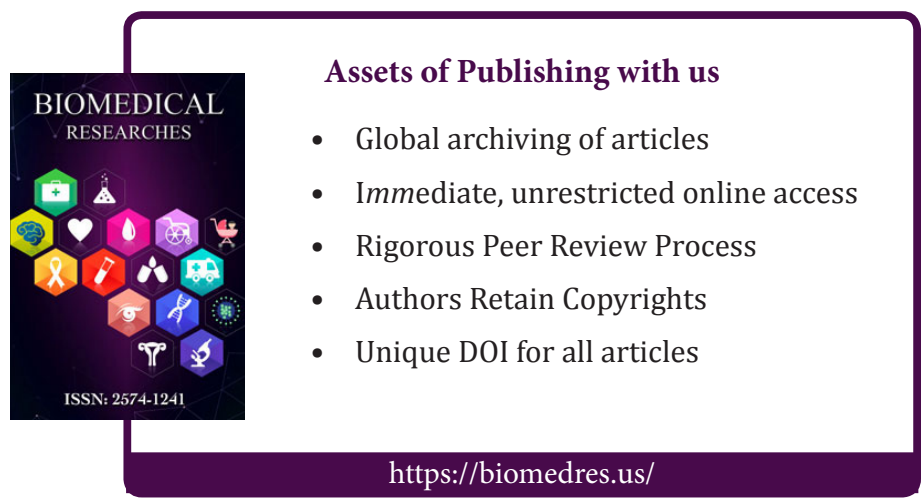

